\title{
Cerenkov Luminescence Imaging for Surgical Margins in Radical Prostatectomy: A Surgical Perspective
}

\author{
Dominic Bagguley ${ }^{1,2}$, Marcus Cumberbatch ${ }^{1,3}$, Nathan Lawrentschuk ${ }^{1,2}$, and Declan G. Murphy ${ }^{1,4}$ \\ ${ }^{I}$ Division of Cancer Surgery, Peter MacCallum Cancer Centre, Melbourne, Victoria, Australia; ${ }^{2}$ E.J. Whitten Prostate Cancer \\ Research Centre, Epworth Healthcare, Richmond, Victoria, Australia; ${ }^{3}$ Academic Urology Unit, University of Sheffield, Sheffield, \\ United Kingdom; and ${ }^{4}$ Sir Peter MacCallum Department of Oncology, University of Melbourne, Parkville, Australia
}

\section{See the associated article on page 1500.}

$\mathbf{H}$ ow can I reduce my positive surgical margin rate?" This is a common refrain from the urologist performing radical prostatectomy (RP) for localized prostate cancer (PC). Avoidance of positive surgical margins (PSMs) during RP is important to reduce the likelihood of biochemical recurrence and the subsequent increased risk of metastases and death from PC (1). PSMs increase the likelihood of receiving additional treatment such as pelvic radiotherapy by up to 5-fold (2), as well as increasing the likelihood of local morbidity and the financial burden of additional treatment. Despite advancements in surgical technologies, a 2017 metaanalysis found that the rate of PSMs for open RP was $31.4 \%$ (range, $21 \%-53 \%$ ), and the rate of PSMs for robot-assisted RP was similar, at $31.7 \%$ (range, $14 \%-50 \%$ ) (3).

Currently, options for surgeons to prevent PSMs are limited and include preoperative and intraoperative strategies. Regarding the former, traditional risk stratification is based on characteristics such as digital rectal examination, multiparametric MRI, biopsy findings, and, more recently, PSMA PET/CT (4). However, these studies are often performed many months before surgery and the true pathology can advance in the interval. Intraoperative techniques such as fresh-frozen sections may be used for intraoperative assessment; however, there remain concerns about high rates of false-negatives (5). Recently, fresh-frozen sections have been incorporated into the NeuroSAFE principle aimed at improving functional outcomes by facilitating preservation of neurovascular bundles when there is uncertainty about a tumor breach into these regions (6). However, we await level 1 evidence to support worldwide use of this method, and limitations in pathology resources and expertise will persist regardless of the data supporting fresh-frozen sections to reduce margins. Therefore, additional strategies to reduce PSM rates are welcome.

\section{PSMA-BASED TECHNOLOGIES IN PC}

${ }^{68} \mathrm{Ga}$-PSMA PET is a powerful imaging modality in PC diagnosis and treatment planning. It relies on antibodies or small molecules to target a particular protein known as prostate-specific membrane antigen

Received Apr. 4, 2020; revision accepted May 27, 2020.

For correspondence or reprints contact: Declan G. Murphy, Division of Cancer Surgery, Peter MacCallum Cancer Centre, 305 Grattan St., Melbourne, Victoria 3002, Australia.

E-mail: declan.murphy@petermac.org

Published online Jun. 26, 2020.

COPYRIGHT (c) 2020 by the Society of Nuclear Medicine and Molecular Imaging. DOI: $10.2967 /$ jnumed.120.243303
(PSMA), found in prostate cells. Because PSMA is expressed in greater concentrations in PC cells, it can be targeted through PET imaging. To enable imaging, the molecules that target PSMA are radiolabeled, often with ${ }^{18} \mathrm{~F}$ or ${ }^{68} \mathrm{Ga}$, and once they have accumulated in PSMA-avid tissue their emissions can be captured by a PET camera. This capability has led to significant advances in the detection of local and metastatic PC in both its primary stage and its biochemically recurrent stage (7). Furthermore, novel applications of PSMA technology are now beginning to emerge and offering to transform intraoperative evaluation of surgical margins in RP (8).

Cerenkov luminescence imaging (CLI) is a technique that was discovered in 2009 and involves the detection of Cerenkov photons (9). Conveniently, these photons are emitted by the same radiolabeled molecules used in PSMA PET $\left({ }^{18} \mathrm{~F}\right.$ and $\left.{ }^{68} \mathrm{Ga}\right)$ and are optically captured and emitted at the same time as the annihilation photons traditionally detected by the PET camera. These images are then overlaid to provide a tool to visually assess the margin status of the tumor specimen $(8,10)$. Although CLI provides only lower-resolution 2-dimensional images, not high-resolution 3-dimensional images, when compared with PET, it is compatible with requirements for image-guided surgery (10). Novel advances have enabled increased portability of CLI systems, giving surgeons real-time, intraoperative CLI-based evaluations of surgical margins in tumor resection (10).

\section{ARTICLE IN FOCUS}

The single-center prospective study by Darr et al. (11) in this issue of The Journal of Nuclear Medicine evaluated the feasibility and accuracy of intraoperative ${ }^{68} \mathrm{Ga}$-PSMA CLI to assess surgical margins in RP. Conducted over 17 mo between 2018 and 2019, the study included 10 men with high-risk PC undergoing RP with no prior history of prostate surgery, known distant metastases, or contraindications to surgery. Initially, ${ }^{68} \mathrm{Ga}$ PSMA PET was performed on subjects 45-60 min after they received an intravenous injection of dose-adjusted ${ }^{68} \mathrm{Ga}$ PSMA-11. After experienced nuclear medicine physicians confirmed that there were no distant metastases, the subjects immediately proceeded to RP with extended pelvic lymph node dissection. This was performed by 2 surgeons to minimize operative time and subsequent radiotracer decay. After removal of the excised prostate, the specimens were placed into the LightPath imaging chamber in the operating room to enable CLI. Images were acquired within $300 \mathrm{~s}$ (a significant improvement on the time taken to obtain results from a fresh-frozen section). Regardless of the CLI results, the surgical course remained unaffected and no further tissue was resected even if the CLI results suggested positive margins. The median time between intravenous injection and start of surgery was $223 \mathrm{~min}$ (range, 153-328 $\mathrm{min}$ ), whereas the time between intravenous 
injection and CLI image acquisition was 333 min (range, 282-429 min). Three of the 10 subjects had PSMs, as is consistent with standard rates of PSMs at RP (1). Two of the $3(66 \%)$ PSMs had been identified by intraoperative CLI, and the cancer identified at the positive margins was graded as International Society of Uropathology groups 4 and 5, with diameters of 2 and $4 \mathrm{~mm}$, respectively. The PSM that was not identified by intraoperative CLI was group 3 PC, supporting preexisting data that have demonstrated reduced PSMA expression on lowergrade PC (12). Finally, only 25 of the 35 regions of interest on CLI demonstrated positive margins by standard histopathology, giving a $28 \%$ false-positive rate. As most of the false positives were seen at the prostate base, Darr et al. suggested contamination by ${ }^{68} \mathrm{Ga}$-containing urine (renally excreted) as a potential explanation for the false-positives. However, the false-positives had PC tissue deep to overlying benign prostatic tissue, suggesting an intrinsic issue with the photon range of the radiotracer rather than urine contamination.

\section{DISCUSSION}

Recent advances in nuclear imaging have enabled the development of relatively cost-effective and portable devices to provide intraoperative guidance regarding surgical margins. Although only in the early stages of integration into clinical practice, intraoperative CLI offers surgeons the possibility of evaluating surgical margins in real time. The device used in the study features a lighttight imaging chamber into which the resected tumor specimen is placed. The specimen is then captured by both CLI and standard white-light imaging. By combining these images, one can see PSMs on tumor specimens, allowing for immediate further surgical resection if required. Of course, a decision to resect more tissue is a significant one, and the current false-positive rate of $28 \%$ reported with CLI in this study means that surgeons are unlikely to further resect on the basis of current data. CLI has been previously described in neurosurgery and breast-conserving surgery (10), and this study was the first of its kind to evaluate CLI during RP for PC.

Although this study was low-powered and designed to demonstrate only the feasibility of intraoperative CLI to reduce PSMs, some interesting observations could be made, and the authors are to be congratulated for this novel work. First, the only PSM missed by CLI had group $3 \mathrm{PC}$ at the surgical margin. Although this is consistent with other studies showing reduced PSMA expression in lower-grade PC (12), the interval between PSMA-agent injection and CLI (median, $333 \mathrm{~min}$ ) was long and potentially detrimental to identification of lower-grade PC. Future studies may aim to reduce the interval between PSMA-agent injection and commencement of surgery to improve signal intensity and potentially the overall sensitivity of CLI. Second, a high number of the false-positives occurred at the base of the prostate. The authors speculated that the presence of radioactive tracer in the urinary bladder (i.e., close to the base of the prostate) might have explained this finding. As such, rinsing the prostate specimen with saline before performing CLI was recommended in future studies. Third, the false PSM was found to have PC tissue deep to the surgical margin, with overlying benign tissue. The authors suggested that using ${ }^{18} \mathrm{~F}$ as the radiotracer instead of ${ }^{68} \mathrm{Ga}$ may reduce the number of false-positive because ${ }^{18} \mathrm{~F}$ has a reduced mean positron range in tissue ( 0.54 vs. $2.83 \mathrm{~mm})$, preventing deeper PC tissue from emitting false-positive signals at benign surgical margins. Nonetheless, even with such proposed improvements, there will always be a potential for CLI signal to identify cancer very close to the edge of the specimen-a situation that may not translate into a PSM on histopathology. These instances will always be considered a false-positive if there is no PSM on histopathology, although there may not be clinical significance. Lastly, like any new technology, there will be a learning curve for surgeons and operating room staff and a need for nuclear medicine physicians, urologists, and pathologists to develop relationships and pathways (13). Part of the challenge will be to overcome any concerns and logistic barriers to the handling of radioactivity in new clinical environments such as the PC operating room.

Of note, in a very recent study of 6 men undergoing RP and CLI using the same LightPath system, the authors reported that CLI correctly identified negative surgical margins and PSMs in 3 of 5 patients (14). One patient was excluded because of unsuccessful labeling on the day of surgery.

In conclusion, whereas Darr et al. (11) present a promising technique for intraoperative assessment of surgical margins in RP within a small sample of patients, further exploration is needed in a larger study with a more clearly defined CLI protocol (currently being undertaken). Furthermore, studies looking at the use of ${ }^{18} \mathrm{~F}$ rather than ${ }^{68} \mathrm{Ga}$ may be helpful to investigate the impact of the former in reducing false-positives in intraoperative CLI.

\section{DISCLOSURE}

No potential conflict of interest relevant to this article was reported.

\section{REFERENCES}

1. Wright JL, Dalkin BL, True LD, et al. Positive surgical margins at radical prostatectomy predict prostate cancer specific mortality. J Urol. 2010;183:2213-2218.

2. Evans SM, Millar JL, Frydenberg M, et al. Positive surgical margins: rate, contributing factors and impact on further treatment-findings from the Prostate Cancer Registry. BJU Int. 2014;114:680-690.

3. Srougi V, Bessa J Jr, Baghdadi M, et al. Surgical method influences specimen margins and biochemical recurrence during radical prostatectomy for high-risk prostate cancer: a systematic review and meta-analysis. World J Urol. 2017;35:1481-1488.

4. Hofman MS, Lawrentschuk N, Francis RJ, et al. Prostate-specific membrane antigen PET-CT in patients with high-risk prostate cancer before curative-intent surgery or radiotherapy (proPSMA): a prospective, randomised, multi-centre study. Lancet. 2020;395:1208-1216.

5. Gillitzer R, Thuroff C, Fandel T, et al. Intraoperative peripheral frozen sections do not significantly affect prognosis after nerve-sparing radical prostatectomy for prostate cancer. BJU Int. 2011;107:755-759.

6. Schlomm T, Tennstedt P, Huxhold C, et al. Neurovascular structure-adjacent frozensection examination (NeuroSAFE) increases nerve-sparing frequency and reduces positive surgical margins in open and robot-assisted laparoscopic radical prostatectomy: experience after 11,069 consecutive patients. Eur Urol. 2012;62:333340.

7. Perera M, Papa N, Roberts M, et al. Gallium-68 prostate-specific membrane antigen positron emission tomography in advanced prostate cancer: updated diagnostic utility, sensitivity, specificity, and distribution of prostate-specific membrane antigen-avid lesions-a systematic review and meta-analysis. Eur Urol. 2020;77:403-417.

8. Grootendorst MR, Cariati M, Kothari A, Tuch DS, Purushotham A. Cerenkov luminescence imaging (CLI) for image-guided cancer surgery. Clin Transl Imaging. 2016;4:353-366.

9. Robertson R, Germanos MS, Li C, Mitchell GS, Cherry SR, Silva MD. Optical imaging of Cerenkov light generation from positron-emitting radiotracers. Phys Med Biol. 2009;54:N355-N365.

10. Ciarrocchi E, Vanhove C, Descamps B, De Lombaerde S, Vandenberghe S, Belcari N. Performance evaluation of the LightPath imaging system for intraoperative Cerenkov luminescence imaging. Phys Med. 2018;52:122-128.

11. Darr C, Harke N, Radtke JP, et al. Intraoperative ${ }^{68}$ Ga-PSMA Cerenkov luminescence imaging for surgical margins in radical prostatectomy: a feasibility study. $\mathrm{J} \mathrm{Nucl} \mathrm{Med}$. 2020;61:1500-1506.

12. Hupe MC, Philippi C, Roth D, et al. Expression of prostate-specific membrane antigen (PSMA) on biopsies is an independent risk stratifier of prostate cancer patients at time of initial diagnosis. Front Oncol. 2018;8:623.

13. Murphy DG, Hofman MS, Azad A, Violet J, Hicks RJ, Lawrentschuk N. Going nuclear: it is time to embed the nuclear medicine physician in the prostate cancer multidisciplinary team. BJU Int. May 4, 2019 [Epub ahead of print].

14. Olde Heuvel J, de Wit-van der Veen BJ, van der Poel HG, et al. ${ }^{68}$ Ga-PSMA Cerenkov luminescence imaging in primary prostate cancer: first-in-man series. Eur J Nucl Med Mol Imaging. April 2, 2020 [Epub ahead of print]. 